\title{
A W-Ne interatomic potential for simulation of neon implantation in tungsten
}

\author{
Marie Backman ${ }^{\mathrm{a}}$, Niklas Juslin ${ }^{\mathrm{a}}$, Guiyang Huang ${ }^{\mathrm{a}}$, Brian D. Wirth ${ }^{\mathrm{a}, \mathrm{b}, *}$ \\ ${ }^{a}$ Department of Nuclear Engineering, University of Tennessee, Knoxville, Tennessee 37996, USA \\ ${ }^{b}$ P.O. Box 2008, MS-6003, Oak Ridge National Laboratory, Oak Ridge, Tennessee 37831, USA
}

\begin{abstract}
An interatomic pair potential for W-Ne is developed for atomistic molecular dynamics simulations of neon implantation in tungsten. The new potential predicts point defect energies and binding energies of small clusters that are in good agreement with electronic structure calculations. Molecular dynamics simulations of small neon clusters in tungsten show that trap mutation, in which an interstitial neon cluster displaces a tungsten atom from its lattice site, occurs for clusters of three or more neon atoms. However, near a free surface, trap mutation can occur at smaller sizes, including even a single neon interstitial in close proximity to a (100) or (110) surface.
\end{abstract}

\section{Introduction}

Tungsten is a candidate material for the divertor in fusion reactors due to its high melting temperature, low plasma fuel retention and low sputtering yield. The divertor will be exposed to fusion plasma exhaust in the form of low energy hydrogen and helium, in addition to heavier impurity gas atoms, causing gas bubbles to form and blistering at the surface. Experiments [1,2] show that exposure of tungsten to low energy helium plasma at temperatures between 1000-2000 K leads to formation of a low density nanostructured surface morphology, called 'fuzz'. The fuzz formation mechanism is not fully understood and needs to be studied to assess how the extreme surface modification affects surface properties and erosion of the divertor.

A recent study at the linear plasma simulator NAGDIS-II [3] showed that fuzz does not form on tungsten under neon or argon plasma exposure in conditions that were otherwise similar to the helium plasma experiments. In this paper, a new interatomic potential for $\mathrm{W}-\mathrm{Ne}$ is presented, which reproduces simple defect energies in excellent agreement with density functional theory (DFT) calculations and makes it possible to study neon implantation in tungsten using atomistic simulations. Using the new potential, we study the reactions of small neon clusters in bulk tungsten and near a tungsten surface by molecular dynamics (MD).

\section{Methods}

\subsection{Potential}

The potential formalism chosen for the W-Ne potential is the same as recently used to describe $\mathrm{W}$-He [4], and which has also been successfully used to describe $\mathrm{Fe}$ and $\mathrm{Cr}$ interactions with $\mathrm{He}[5,6]$. Interaction potentials from the literature were used to describe the two other possible interactions, W-W and Ne-Ne. The W-W potential is of the Finnis-Sinclair N-body formalism [7] as modified by Ackland and Thetford [8] and more recently modified by Juslin et al. [4] for cascade simulations by adding a short range fit to the Ziegler-Biersack-Littmark (ZBL) potential [9]. The Ne-Ne interactions were modeled using the pair potential by Aziz [10].

\footnotetext{
* Corresponding author

Email address: bdwirth@utk.edu (Brian D. Wirth)
} 
The molecular dynamics simulations using these potentials were performed with the simulation code LAMMPS [11], which provides a "hybrid" potential style with which different potential formalisms can be combined. The potential energy of a neon atom is thus given by a sum of contributions from tungsten and neon neighbors:

$$
E_{i}^{\mathrm{Ne}}=\sum_{\mathrm{W} \text { neighb }} V_{\mathrm{W}-\mathrm{Ne}}\left(r_{i j}\right)+\sum_{\mathrm{Ne} \text { neighb }} V_{\mathrm{Ne}-\mathrm{Ne}}\left(r_{i j}\right)
$$

The energy of a tungsten atom is given by pair potential contributions from neon and tungsten neighbors, as well as an embedding function, $\phi_{\mathrm{W}-\mathrm{W}}$, which depends on the number and distance of the neighboring tungsten atoms, as:

$$
E_{i}^{\mathrm{W}}=\sum_{\text {Ne neighb }} V_{\mathrm{W}-\mathrm{Ne}}\left(r_{i j}\right)+\sum_{\mathrm{W} \text { neighb }} V_{\mathrm{W}-\mathrm{W}}\left(r_{i j}\right)+\sum_{\mathrm{W} \text { neighb }} \phi_{\mathrm{W}-\mathrm{W}}\left(r_{i j}\right)
$$

LAMMPS automatically performs the summation of contributions from different potentials. The potential functions $V_{\mathrm{Ne}-\mathrm{Ne}}, V_{\mathrm{W}-\mathrm{W}}$, and $\phi_{\mathrm{W}-\mathrm{W}}$ were taken from the literature, as described above, and a new potential for $\mathrm{W}-\mathrm{Ne}, V_{\mathrm{W}-\mathrm{Ne}}$, is presented here.

To correctly describe the strong repulsion at short ranges, the W-Ne potential developed here was also joined with the ZBL potential at a distance much shorter than the nearest-neighbor distance, and the resulting potential is given as:

$$
V_{W-N e}\left(r_{i j}\right)= \begin{cases}\mathrm{ZBL}, & r_{i j} \leq r_{1} \\ p_{5} r_{i j}^{5}+p_{4} r_{i j}^{4}+p_{3} r_{i j}^{3}+p_{2} r_{i j}^{2}+p_{1} r_{i j}+p_{0}, & r_{1} \leq r_{i j} \leq r_{2} \\ \left(a+\frac{b}{r_{i j}}\right) e^{-c r_{i j}} f_{c}\left(r_{i j}\right) & r_{i j} \geq r_{2}\end{cases}
$$

The cutoff function $f_{c}\left(r_{i j}\right)$ makes the potential go smoothly to zero at the chosen cutoff distance, and it is given by

$$
f_{c}\left(r_{i j}\right)= \begin{cases}1, & r_{i j} \leq r_{c}-r_{d} \\ \frac{1}{2}\left(1-\sin \frac{\pi\left(r_{i j}-r_{c}\right)}{2 r_{d}}\right), & \left|r_{c}-r_{i j}\right| \leq r_{d} \\ 0, & r_{i j} \geq r_{c}+r_{d}\end{cases}
$$

\subsection{Atomistic simulations}

MD simulations were performed with LAMMPS to study defect formation energies and binding energies of small clusters. The simulation cell consisted of a body-centered cubic (bcc) lattice $20 \times 20 \times 20$ lattice units in size (16,000 atoms) and periodic boundary conditions were applied. To calculate binding energies of small neon clusters, the simulation cells were first relaxed at $1000 \mathrm{~K}$ for $20 \mathrm{ps}$ and then the temperature was slowly decreased to $0 \mathrm{~K}$ using a Berendsen thermostat in LAMMPS. The pressure in the simulation cell, due to the decrease in temperature under constant volume, was subsequently released by applying a Nose-Hoover thermostat and barostat. The same simulation methods were used to determine the critical cluster size for bulk trap mutation. It is possible that the cluster ground state was not reached with this method and the clusters should probably be considered meta-stable states.

The formation energy of a defect is defined as

$$
E_{f}=E_{\text {def }}-E_{\text {ref }} \cdot\left(N-m_{v a c}\right) / N+E_{r e f, N e} \cdot n_{N e}
$$

where $E_{\text {def }}$ is the total energy of the system including the defect, $E_{\text {ref }}$ is the total energy of the system without the defect (pure bcc tungsten), $\mathrm{N}$ is the number of atoms in the pure tungsten simulation cell, and $m_{v a c}$ is the number of vacancies in the system containing the defect. The reference energy $E_{r e f, N e}$ is calculated as the cohesive energy of close-packed neon, which for the Aziz potential is $-0.02446 \mathrm{eV} / \mathrm{atom}$.

Binding energies of small Ne clusters were calculated using the expression 
Table 1: Parameters for the new W-Ne potential.

\begin{tabular}{cccc}
$a$ & -36.5 & $p_{0}$ & $1.00398371 \mathrm{e}+04$ \\
$b$ & 293.86 & $p_{1}$ & $-3.73197023 \mathrm{e}+04$ \\
$c$ & 1.9 & $p_{2}$ & $5.76706441 \mathrm{e}+04$ \\
$r_{1}$ & 0.80 & $p_{3}$ & $-4.54738275 \mathrm{e}+04$ \\
$r_{2}$ & 1.50 & $p_{4}$ & $1.80689841 \mathrm{e}+04$ \\
$r_{c}$ & 2.85 & $p_{5}$ & $-2.87210879 \mathrm{e}+03$ \\
$r_{d}$ & 0.25 & & \\
\hline
\end{tabular}

$$
E_{b}\left(A_{1}, A_{2}\right)=E\left(A_{1}\right)+E\left(A_{2}\right)-\left(E\left(A_{1}+A_{2}\right)+E_{r e f}\right)
$$

where $A_{1}$ and $A_{2}$ are different defects, $A_{1}+A_{2}$ is the combination of the two individual defects, and $E_{\text {ref }}$ is defined as for equation 5. For the binding energy of a neon atom to a cluster of size $m, A_{1}$ would be a single neon interstitial, $A_{2}$ the cluster that the neon atom is being added to, and $A_{1}+A_{2}$ a cluster of size $m+1$.

To study the reactions of neon impurities near tungsten surfaces, we used a simulation cell of approximately 16,000 atoms with a free surface in either the (100) or the (110) direction. Periodic boundary conditions were applied in two directions, leaving free surfaces in the third direction. The top surface was free while the bottom surface had three layers of fixed atoms to prevent drift of the simulation box. Simulations were performed at $10 \mathrm{~K}$ or $1000 \mathrm{~K}$.

At $1000 \mathrm{~K}$, only neon single and di-interstitials were simulated. The interstitials were placed 10 atom layers below the surface and ten independent simulations were run until the interstitial reached the free surface. At $10 \mathrm{~K}$, no interstitials are mobile and we simulated the evolution of small clusters as a function of depth below the surface. As a starting point for the minimization step, 3 atom clusters were planar and 4 atom clusters were placed in a tetrahedral configuration. The neon atoms in the clusters were placed in adjacent tetrahedral interstitial positions in the plane between atom layers, except for the clusters containing 4 atoms, for which the center of mass was in the next atom plane. Ten statistically independent simulations were run for each combination of cluster size and depth below the surface. The end state with the lowest potential energy was chosen as representative of the ground state. The end states of the neon clusters were categorized either as desorbed, trap mutated, or interstitial. The clusters were labeled desorbed only if all the atoms in the cluster were desorbed.

\section{Results}

\subsection{Potential}

The parameters of the potential formalism as shown in Eq. 3 were fit to DFT data at bond distances relevant for quasi-equilibrium simulations. The data used for the fit is a set of results by Huang et al. that was a first version of the results published in Ref. [12]. Both sets of DFT results are shown in Table 2. The potential was fit to the defect properties shown in Table 2, namely, the formation energies of a substitutional neon atom and of neon interstitials in tetrahedral or octahedral positions in tungsten, as well as the self-binding energy and the migration energy of neon in tungsten. The parameters are shown in Table 1 and the potential is plotted in Fig. 1.

Table 2 shows a comparison between the defect formation energies in the new pair potential and in DFT calculations by Huang et al. [12]. The defect formation energies agree well with the DFT values and, in particular, the relative order of the formation energies is the same. The energy difference between octahedral and tetrahedral interstitials is smaller for this potential than the published DFT results, but the neon interstitial migration energy remains below $0.2 \mathrm{eV}$, indicative of a fast diffusing species. The potential was developed for use with the Ackland tungsten potential and, hence, the results presented in Table 2 are valid only for the combination of potentials used in this work. 


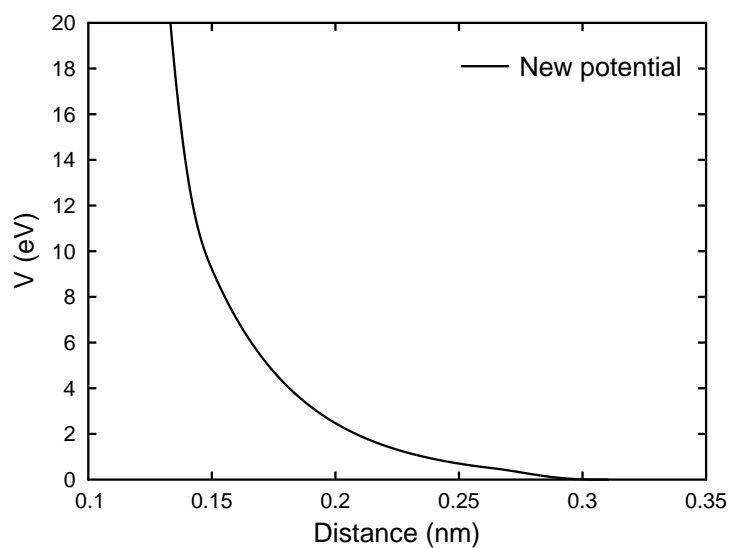

Figure 1: The new W-Ne pair potential.

Table 2: Comparison of the new W-Ne potential and DFT data for the formation energy of substitutional neon and interstitial neon in the tetrahedral and octahedral positions, as well as the Ne-Ne binding energy and the migration energy between two tetrahedral positions. Energies are in $\mathrm{eV}$.

\begin{tabular}{ccc}
$\begin{array}{c}\text { Potential } \\
\text { (this work) }\end{array}$ & $\begin{array}{c}\text { DFT (initial, } \\
\text { unpublished fitting data) }\end{array}$ & $\begin{array}{c}\text { DFT } \\
\text { (published) }\end{array}$ \\
\hline 5.96 & 6.17 & 6.42 \\
11.17 & 11.14 & 11.40 \\
11.30 & 11.29 & 11.95 \\
2.02 & 2.23 & 2.28 \\
0.17 & 0.15 & 0.15 \\
\hline
\end{tabular}


Table 3: Comparison of neon binding energy to interstitial neon and neon-vacancy clusters, as a function of the number of neon in the cluster, using MD simulations with the new W-Ne potential and DFT calculations. Energies are in eV. In the MD cases marked by an asterisk, trap mutation occurred.

\begin{tabular}{lcccc}
\hline & \multicolumn{2}{c}{$0 \mathrm{~V}$} & \multicolumn{2}{c}{$1 \mathrm{~V}$} \\
& Pot. & DFT & Pot. & DFT \\
\hline $0 \mathrm{Ne}$ & & & 8.85 & 8.23 \\
$1 \mathrm{Ne}$ & 2.02 & 2.28 & 3.94 & 4.07 \\
$2 \mathrm{Ne}$ & $3.23^{*}$ & 2.79 & 4.25 & 3.76 \\
$3 \mathrm{Ne}$ & $4.68^{*}$ & 3.71 & 4.74 & 4.89 \\
\hline
\end{tabular}

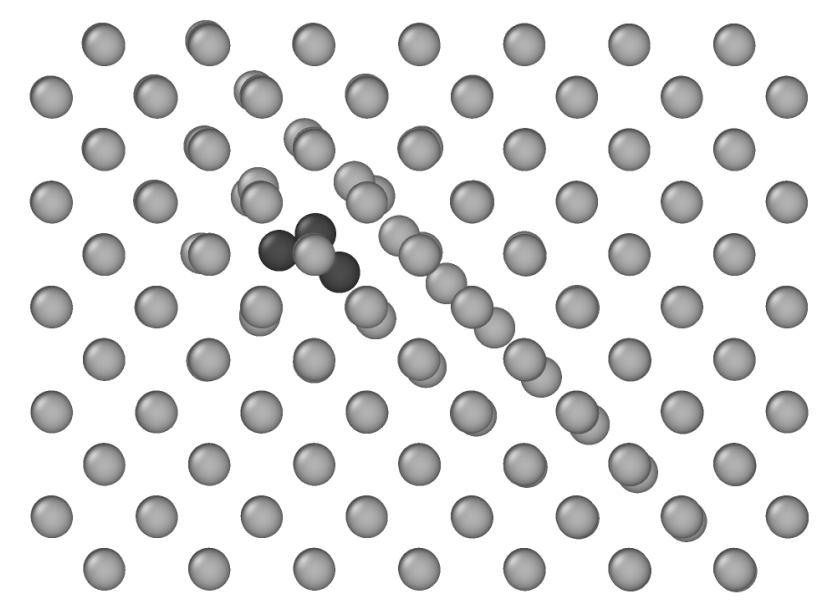

Figure 2: A neon cluster with three atoms occupying a substitutional position in the tungsten lattice. This is the relaxed configuration after thermalizing the simulation box at $1000 \mathrm{~K}$ and slowly decreasing the temperature to $0 \mathrm{~K}$. In the vicinity of the cluster, a crowdion has formed in the (111) direction to accommodate the extra tungsten atom.

In addition to the formation energies of single interstitials, which were part of the fitting database, we compared the defect binding energies of a neon atom to a vacancy or an existing neon cluster to DFT results by Huang et al. [12]. The results are shown in Table 3. The results for a neon atom binding to a vacancy or to a single neon atom (whether interstitial or substitutional) show good agreement with the DFT data. Notably, the MD simulations predict trap mutation to occur for neon interstitial clusters of three or more atoms. In the DFT calculations, the clusters were assumed to be interstitial and would in general not undergo trap mutation unless the activation energy was very low. It is therefore consistent that the binding energies to interstitial clusters of two or three atoms differ between MD and DFT. It is likely that also the atomic configurations of the neon-vacancy clusters differ to some extent between the two methods, especially for clusters of three or four neon atoms, which can explain the discrepancy in binding energies even in the absence of trap mutation.

The cluster relaxation simulations thus showed that the critical size for trapmutation using the MD potential is three neon atoms in a cluster. As a reference point, helium clusters undergo trap mutation at cluster sizes from about 7-8 atoms [13] due to their relatively smaller atomic size. The configuration at $0 \mathrm{~K}$ is shown in Fig. 2, where the $\mathrm{Ne}_{3}$ occupies a substitutional position in the tungsten lattice and the additional tungsten has led to the formation of a crowdion in the (111) direction.

Another interatomic potential for W-Ne [14], based on the EAM formalism, was recently published by Zhou et al. for use with the tungsten EAM potential by Marinica et al. [15]. The formation energies and binding energies agree well between the two potentials. Zhou et al. determine binding energies to neon clusters up to four atoms in size, but it is not clear whether trap mutation occurs in their simulations. 


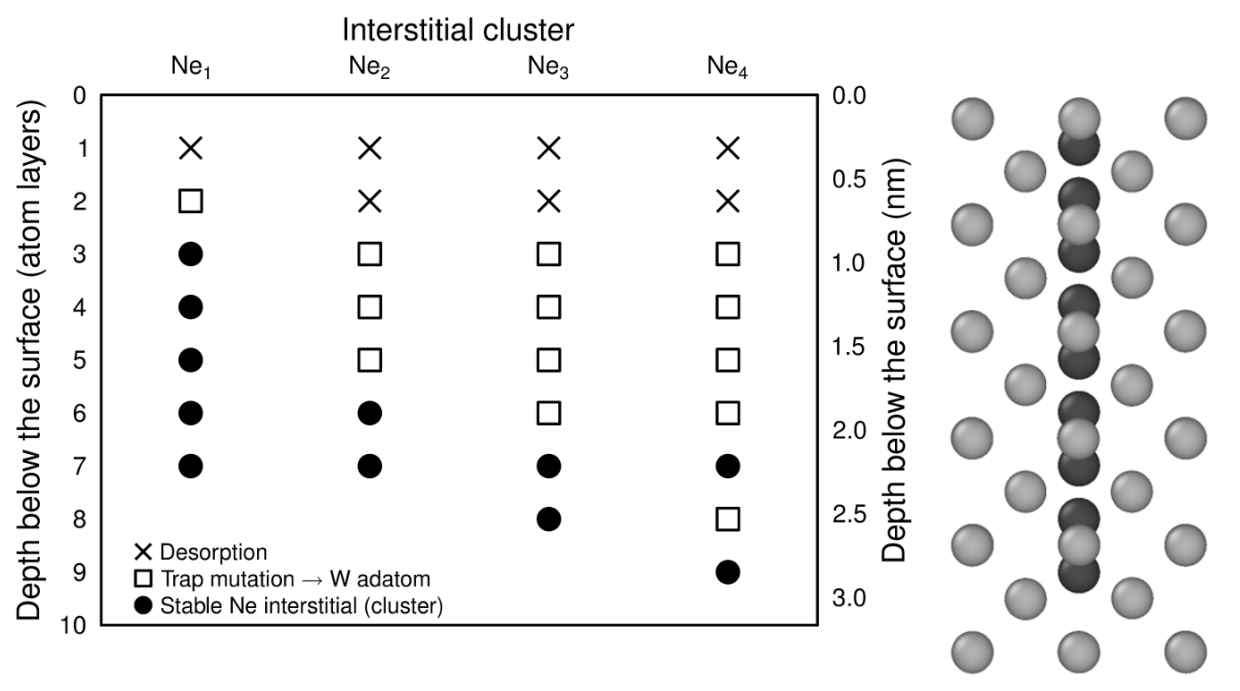

Figure 3: Results of MD simulations of small neon clusters near a (100) tungsten surface (left). The depth below the surface indicates below which atom layer the center of mass of the cluster was positioned. The distance between layers is 0.158 nm. The positioning of the interstitials is shown in the atomistic picture (right).

\subsection{Dynamics of neon clusters near tungsten surfaces}

Close to a free surface, we found that trap mutation occurs at even smaller sized neon clusters. Simulations were performed at $10 \mathrm{~K}$ for a (100) and a (110) surface for cluster sizes up to 4 neon atoms. For neon clusters initially placed within one to two layers below the surface, the neon clusters either immediately desorbed, or displaced tungsten atoms from their lattice site to become substitutional atoms in a trap mutation reaction. The latter reaction can be written $\mathrm{W}+\mathrm{Ne} \rightarrow \mathrm{Ne}-\mathrm{V}+\mathrm{W}_{\mathrm{s}}$, where $\mathrm{V}$ is a vacancy and $\mathrm{W}_{\mathrm{s}}$ is a tungsten adatom. Farther from the surface, clusters of this size remain as interstitials. The simulation results for different cluster sizes as a function of initial depth below the surface are shown in Figures 3 and 4 for a (100) and (110) surface, respectively. Depending on the cluster size, the surface effects disappear after between 3 to 8 atom layers, with the influence of the surface reaching deeper for larger clusters. The trends are similar for the (100) and (110) surfaces.

Since the clusters near surfaces are by design meta-stable states, the evolution of the system is governed by small perturbations and the resulting trends should be seen as approximate. (Such meta-stable clusters could be formed for instance by low-energy neon implantation in tungsten.) For instance, the result that $\mathrm{Ne}_{4}$ clusters below the 8th layer undergo trap mutation while the same cluster below the 7th layer forms a stable interstitial likely only points to the limited number of statistically independent simulations performed to find the ground state.

It should be noted that below the region close to the surface, clusters of three or four neon atoms formed stable interstitial clusters at $10 \mathrm{~K}$, despite the fact that trap mutation would lead to a lower energy state, as evidenced by the relaxation simulations at $1000 \mathrm{~K}$. Trap mutation thus requires an activation energy that is available at the higher temperature but not at the lower. To ensure that the behavior seen at $10 \mathrm{~K}$ was in fact due to the lower temperature and not related to the presence of the free surface, we performed simulations of clusters close to a free surface in which the tungsten atoms were thermalized at $1000 \mathrm{~K}$. At the higher temperature, the region in which the clusters interacted with the surface reached deeper, but below that region, clusters of three or four neon atoms underwent trap mutation to form a tungsten interstitial as expected.

High-temperature simulations at $1000 \mathrm{~K}$ showed that neon single and di-interstitials were highly mobile in tungsten. Neon clusters are thus trapped at a lower number of interstitial atoms than helium clusters in tungsten, which using MD simulations have been predicted to be mobile up to a size of 7 atoms at 


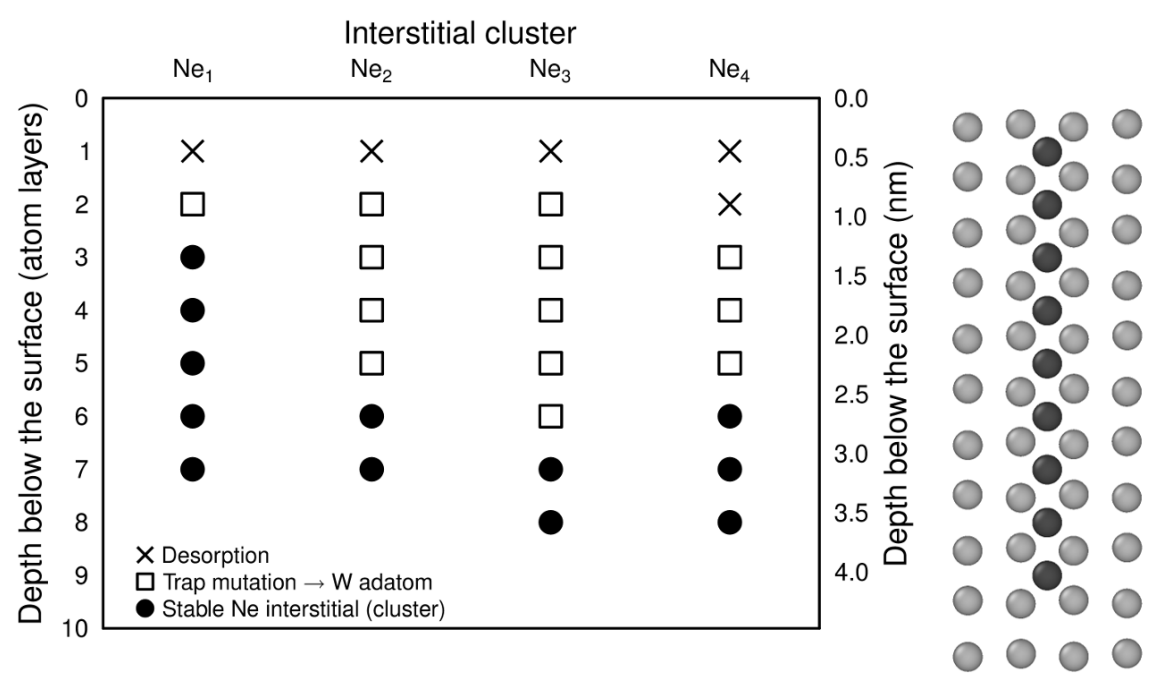

Figure 4: Results of MD simulations of small neon clusters near a (110) tungsten surface (left). The depth below the surface indicates below which atom layer the center of mass of the cluster was positioned. The distance between layers is $0.224 \mathrm{~nm}$. The positioning of the interstitials is shown in the atomistic picture (right).

$1200 \mathrm{~K}$ [13]. In all simulations, the single and di-interstitials underwent trap mutation when reaching the surface. At the (100) surface, single interstitials occupied substitutional positions in the 3rd atom layer, while displacing a single tungsten atom to the surface. Di-interstitials were most likely to be trapped in the 5 th atom layer below the surface. At the (110) surface, trap mutation occurred in the 3rd or 4th atom layer for single interstitials and in the 5th layer for di-interstitials.

MD simulations of small helium clusters near tungsten surfaces $[16,17]$ showed that trap mutation, with or without cluster dissociation, is the dominant mechanism for mobile clusters (up to $7 \mathrm{He}$ atoms). Neon clusters undergo trap mutation at a smaller cluster size, but trap mutation is the most important interaction mechanism. Compared to helium, neon is thus less mobile in tungsten. We will explore further the differences between neon and helium implantation in tungsten in a forthcoming paper.

\section{Conclusions}

A new interatomic potential for W-Ne was developed. Simple defect formation energies are in good agreement with recent DFT results. The new potential was used in molecular dynamics simulations to study the reactions of of small neon clusters in tungsten. Trap mutation occurred for clusters of three neon atoms in the bulk, while a single neon interstitial was sufficient to cause trap mutation near a surface.

\section{Acknowledgements}

Partial financial support for this work was provided through the Scientific Discovery through Advanced Computing (SciDAC) project on Plasma-Surface Interactions, funded by the U. S. Department of Energy, Offices of Science, Advanced Scientific Computing Research, and Fusion Energy Sciences. Additional funding was provided through the Plasma-Surface Interactions Science Center, funded by the U. S. Department of Energy, Office of Fusion Energy Sciences under award DE-SC00-02060 and by the U.S. Department of Energy, Office of Fusion Energy Sciences under award DE-SC0006661. 


\section{References}

[1] M. Baldwin, R. Doerner, Helium induced nanoscopic morphology on tungsten under fusion relevant plasma conditions, Nuclear Fusion 48 (3) (2008) 035001.

URL http://stacks.iop.org/0029-5515/48/i=3/a=035001

[2] S. Kajita, W. Sakaguchi, N. Ohno, N. Yoshida, T. Saeki, Formation process of tungsten nanostructure by the exposure to helium plasma under fusion relevant plasma conditions, Nuclear Fusion 49 (9) (2009) 095005. URL http://stacks.iop.org/0029-5515/49/i=9/a=095005

[3] M. Yajima, M. Yamagiwa, S. Kajita, N. Ohno, M. Tokitani, A. Takayama, S. Saito, A. M. Ito, H. Nakamura, N. Yoshida, Comparison of damages on tungsten surface exposed to noble gas plasmas, Plasma Science and Technology 15 (3) (2013) 282. URL http://stacks.iop.org/1009-0630/15/i=3/a=18

[4] N. Juslin, B. Wirth, Interatomic potentials for simulation of He bubble formation in W, Journal of Nuclear Materials 432 (1-3) (2013) 61-66. doi:10.1016/j.jnucmat.2012.07.023. URL http://linkinghub.elsevier.com/retrieve/pii/ S0022311512003820

[5] N. Juslin, K. Nordlund, Pair potential for Fe-He, Journal of Nuclear Materials 382 (2-3) (2008) $143-146$. doi:10.1016/j.jnucmat.2008.08.029.

URL http://linkinghub.elsevier.com/retrieve/pii/ S0022311508004558

[6] D. Terentyev, N. Juslin, K. Nordlund, N. Sandberg, Fast three dimensional migration of He clusters in bcc Fe and Fe-Cr alloys, Journal of Applied Physics 105 (10) (2009) 103509. doi:10.1063/1.3126709.

URL http://scitation.aip.org/content/aip/journal/jap/105/10/10.1063/1.3126709

[7] M. Finnis, J. Sinclair, A simple empirical N-body potential for transition metals, Philosophical Magazine A 50 (1) (1984) 45-55. doi:10.1080/01418618408244210.

URL http://www.tandfonline.com/doi/abs/10.1080/01418618408244210

[8] G. Ackland, R. Thetford, An improved N-body semi-empirical model for body-centred cubic transition metals, Philosophical Magazine A 56 (1) (1987) 15-30. doi:10.1080/01418618708204464. URL http://dx.doi.org/10.1080/01418618708204464

[9] J. Ziegler, J. Biersack, U. Littmark, The stopping and range of ions in solids, Pergamon, New York, 1985.

[10] R. Aziz, M. Slaman, The Ne-Ne interatomic potential revisited, Chemical Physics 130 (1-3) (1989) 187 - 194. doi:http://dx.doi.org/10.1016/0301-0104(89)87048-X. URL http://www.sciencedirect.com/science/article/pii/ 030101048987048X

[11] S. Plimpton, Fast parallel algorithms for short-range molecular dynamics, Journal of Computational Physics 117 (1) (1995) 1-19. doi:10.1006/jcph.1995.1039.

URL http://linkinghub.elsevier.com/retrieve/pii/ S002199918571039X

[12] G. Huang, N. Juslin, B. D. Wirth, First-principles study of vacancy, interstitial, noble gas atom interstitial and vacancy clusters in W, submitted to J. Appl. Phys.

[13] F. Sefta, K. D. Hammond, N. Juslin, B. D. Wirth, Tungsten surface evolution by helium bubble nucleation, growth and rupture, Nuclear Fusion 53 (7) (2013) 073015. doi:10.1088/0029-5515/53/7/073015. URL http://stacks.iop.org/0029-5515/53/i=7/ a=073015?key=crossref.6dd0a1535bd104bd5fc2bf853655894a

[14] F. Zhou, J. Fang, H. Deng, J. Liu, S. Xiao, X. Shu, F. Gao, W. Hu, New interatomic potentials for studying the behavior of noble gas atoms in tungsten, Journal of Nuclear Materials 467 (2015) 398-405. doi:10.1016/j.jnucmat.2015.05.004. URL http://linkinghub.elsevier.com/retrieve/pii/ S0022311515002688

[15] M.-C. Marinica, L. Ventelon, M. R. Gilbert, L. Proville, S. L. Dudarev, J. Marian, G. Bencteux, F. Willaime, Interatomic potentials for modelling radiation defects and dislocations in tungsten, Journal of Physics: Condensed Matter 25 (39) (2013) 395502. doi:10.1088/0953-8984/25/39/395502.

URL http://stacks.iop.org/0953-8984/25/i=39/a=395502?key=crossref.a4fd641b098493a4a64b9c86d03d1990

[16] L. Hu, K. D. Hammond, B. D. Wirth, D. Maroudas, Dynamics of small mobile helium clusters near tungsten surfaces, Surface Science 626 (2014) L21-L25. doi:10.1016/j.susc.2014.03.020. URL http://linkinghub.elsevier.com/retrieve/pii/ S0039602814000867

[17] L. Hu, K. D. Hammond, B. D. Wirth, D. Maroudas, Interactions of mobile helium clusters with surfaces and grain boundaries of plasma-exposed tungsten, Journal of Applied Physics 115 (17) (2014) 173512. doi:10.1063/1.4874675. URL http://scitation.aip.org/content/aip/journal/jap/115/17/ 10.1063/1.4874675 